\title{
Paper
}

\section{Research on High Efficiency and Optimization of Compact Wind Power Generation System applying "Mahalanobis-Taguchi-System"}

\author{
EIJI TOMA ${ }^{* \dagger} \quad$ Member, $\quad$ Hiroshi TANAKA ${ }^{\ddagger}$ Non-member \\ KAZUSHIGE KIKUTA ${ }^{\dagger}$ Non-member
}

(Received September 25, 2018, revised December 19, 2018)

\begin{abstract}
While global reinforcement of countermeasures against global warming is required, Japan depends on overseas imports for over $95 \%$ of energy consumption. From the resource production site to the domestic final consumption, it is important to continuously build a stable energy supply system. With the accident at TEPCO Fukushima nuclear power plant occurred on March 11, 2011, the expectation for renewable energy is rapidly rising. Among them, the eyes to wind power generation are changing in particular. At the end of 2012 the amount of wind power generation equipment installed worldwide corresponds to 280 nuclear power plants. National Institute of Technology Tsuruoka College introduced a fully independent power supply system by wind power as a part of human resource development project for engineers. In this research, we were able to extract the task by analyzing the power generation characteristics applying "Mahalanobis-Taguchi-System (MTS)", diagnosing the cause and optimizing power generation efficiency.
\end{abstract}

Keywords: Wind power generation, MT system, Power generation efficiency, Mahalanobis distance (MD), SN ratio.

\section{Introduction}

While global reinforcement of countermeasures against global warming is required, Japan now depends on overseas imports for more than $95 \%$ of energy consumption. Continuously building a stable energy supply system from resource production areas to domestic final consumption is an important issue and from the viewpoint of securing such energy security it is essential to maintain renewable energy Expansion of popularization is indispensable. In the "New Growth Strategy" decided by the Cabinet in June 2010, "Global Innovation Environment and Energy Power Strategy" was listed as a pillar of the seven strategic fields. Among them, the importance of expanding the spread of renewable energy and constructing a smart grid supporting it has been demonstrated. As a result of a power outage continued at the Great Tsunami caused by the Great East Japan Earthquake that occurred on March 11, 2011, a series of nuclear accidents such as hydrogen explosion and meltdown occurred at the Fukushima Daiichi Nuclear Power Station, and radioactivity was scattered over a wide area. In response to this accident, further stringent safety measures are required, and it is expected that countermeasure and administrative costs will increase accordingly [1]. In that situation, the eyes to wind power generation are changing. At the end of 2012 the amount of wind power generation equip-

\footnotetext{
* Corresponding author: toma-e@tomakomai-ct.ac.jp

$\dagger$ National Institute of Technology, Tomakomai College,

442, Tomakomai, Nishikioka, Hokkaido, Japan, 059-1275

$¥$ Aichi Institute of Technology,

1247, Yachikusa, Yakusa, Toyota, Aichi, Japan, 470-0392
}

ment installed worldwide corresponds to 280 nuclear power plants. However, among the electricity generated in Japan, the share of wind power generation is only $0.4 \%$ in 2010 . Although Japan is most delayed in developed countries, the potential of wind power generation is high, especially in Hokkaido and Tohoku areas, it is said that there is a potential to exceed the power generation capacity of Tohoku Electric Power [2] [12]. In this research, we will consider wind power generation, which is the mainstay of renewable energy which is being introduced worldwide alongside solar power generation. National Institute of Technology Tsuruoka College introduced a fully independent power supply system by wind power as a part of human resource development project for engineers [13]. The total power generation of the wind-generated system is $1 \mathrm{~kW}$ (at $12.5 \mathrm{~m} / \mathrm{s}$ ) and The main body weight is $18 \mathrm{~kg}$.

We have worked on optimization of power generation efficiency (high efficiency and output stabilization) by analysis of power generation characteristics applying "Mahalanobis-Taguchi-System (MTS)" to introduced wind power generation and cause diagnosis by application of parameter design.

\section{Technical problem}

Wind power generation is used by converting wind energy, which is natural energy, into electric energy. The characteristic of wind power generation is that it is a clean power generation with no emission of environmental pollutants such as carbon dioxide and radioactive material, because it uses renewable energy of wind, and energy resources are almost 
Table 1: Main specifications

\begin{tabular}{l|l}
\hline Items & Specifications \\
\hline \hline Windmill type & Propeller type \\
\hline Rotor diameter & $1800 \mathrm{~mm}$ \\
\hline Blades number & 3 pieces \\
\hline Generator type & Synchronization \\
\hline Control system & $\begin{array}{c}\cdot \text { Power assist } \\
\cdot \text { Posture control }\end{array}$ \\
\hline Wind velocity to cut in & $2.5 \mathrm{~m} / \mathrm{s}$ \\
\hline Rated output & $1 \mathrm{~kW}(12.5 \mathrm{~m} / \mathrm{s})$ \\
\hline Rated revolutions & $1000 \mathrm{rpm}$ \\
\hline
\end{tabular}

inexhaustible. However, since the wind constantly changes and the wind direction and the wind speed constantly fluctuate, it is difficult to obtain a stable power generation output, and the wind energy density is small. The function of wind power generation is not to simply rotate. It must be a device that rotates more smoothly and efficiently converts from its rotation to electrical energy. Also, it is required that stable electric power can be generated against environmental conditions and aged deterioration of places to be installed. National Institute of Technology Tsuruoka College introduced a fully independent power supply system by wind power as a part of human resource development project for engineers. Conventionally, at a certain rotation speed of a generator, the wind speed and the power generation amount (output) are in a proportional relationship, but when it exceeds a certain win speed, there is a problem that the efficiency decreases and the output varies. In this research, we extracted factors of these problems and analyzed the characteristics of power generation efficiency and amount by applying MTS, and approached high efficiency and optimization of power generation amount [11].

2.1 System analysis The main specifications and external view of the independent power wind power generation system (AIRDOLPHIN Zero : Zephyr Corporation) introduced in this research are shown in Table 1 and Fig. 1. Fig. 2 shows the relation between the wind speed measured at the time of system introduction (start of operation) and a certain period of time (diagnosis target period) after introduction. In the experiment method, output data (wind speed, rotor rotation speed, power generation amount, etc.) in a certain period from the measurement control unit of the generator was recorded in the PC.

"Base curve" shows the output characteristics at the time of system introduction (at the start of operation), and "target curve" shows the output characteristics for a certain period (diagnosis target period) after introduction. "Standard curve" is the standard output characteristic described in the catalog of the introduced wind power generation system. The verification results of the output characteristics (power curve) of the wind power generation system are shown in Table 2. The wind turbine output data in the operation start period was taken as unit data and the output data in the diagnosis target period was taken as signal data. The fluctuation rate of $\pm 12.5 \%$ is the fluctuation rate of the output of the measured power generation amount. As can be seen from

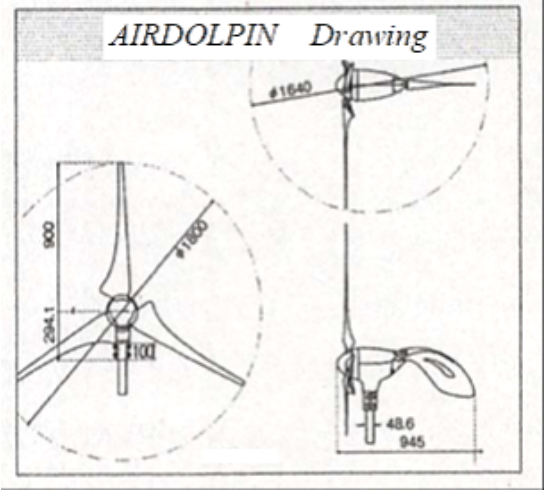

Figure 1: External view

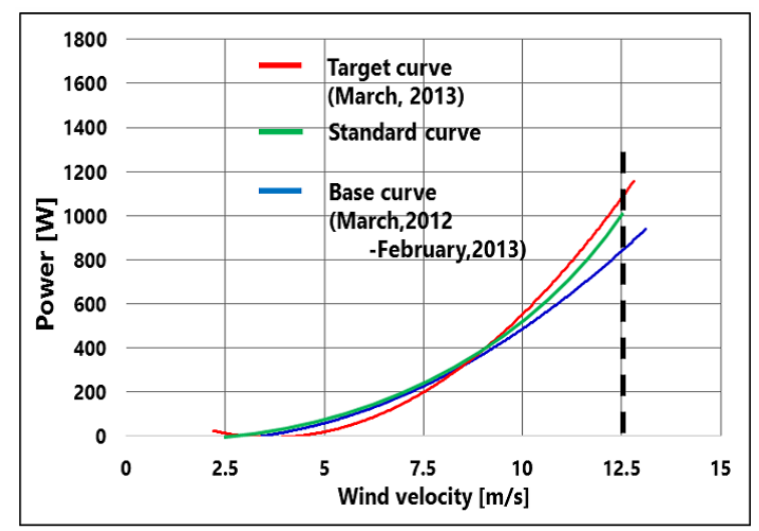

Figure 2: Measurement result of output properties

Table 2: Output properties comparison

\begin{tabular}{l|c|c|c}
\hline & $\begin{array}{c}\text { Wind } \\
\text { velocity }[\mathrm{m} / \mathrm{s}]\end{array}$ & Power $[\mathrm{W}]$ & $\begin{array}{c}\text { Output } \\
\text { regulation }\end{array}$ \\
\hline \hline Standard curve & 12.5 & 1000 & \multirow{2}{*}{ $\pm 12.5 \%$} \\
\hline Base curve & 12.5 & 900 & \multirow{2}{*}{ Target curve } \\
\hline
\end{tabular}

Fig. 2 and Table.2, the fluctuation of $\pm 12.5 \%$ ( $\max$ ) is observed in the output fluctuation rate in the wind speed range of 9 to $12.5 \mathrm{~m} / \mathrm{s}$.

2.2 Application of "MTS" "Mahalanobis-TaguchiSystem (MTS)" is a new method of pattern recognition and has been applied to a wide range of science and technology fields such as health diagnosis, appearance inspection, abnormality monitoring, characteristics estimation of material blending, price estimation of real estate, etc. P.C. Mahalanobis (1893 - 1972), a prominent statistician in India who founded the Indian Institute of Statistical Research, devised "Mahalanobis Distance (MD)" considering the correlation between items [3]. In Japan, research of MTS began by Dr. G. Taguchi (1924 - 2012) from the latter half of 1970's. MTS is a pattern recognition method that incorporates MD into the "Taguchi method" system, and is a general purpose technique to integrate multiple measurement values into one sort. Pattern recognition is information processing technology that recognizes and predicts based on various information obtained from objects. Along with faster computer processing and higher memory capacity, it became possible to 


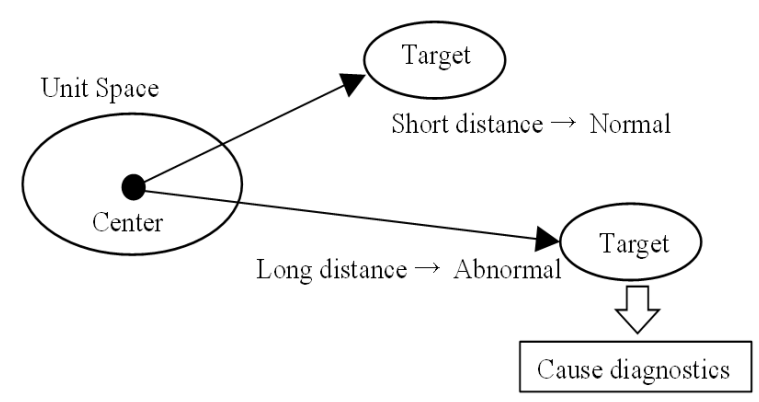

Figure 3: Conceptual drawing

solve pattern recognition handling a large amount of data which was impossible up to now. Application of MTS can greatly contribute to the field of manufacturing and environmental problems. Renewable energy technologies such as nuclear power generation, wind power generation, solar power generation and the like are likely to suffer from inexperienced troubles. MTS is a technology that prevents accidents and defects, since it can detect symptoms of abnormality even for inexperienced troubles [4].

2.3 Features of "MTS" MTS is a method of defining a normal pattern of target multidimensional data as a unit space and obtaining data outside the unit space (signal space) as a distance corresponding to the unit quantity of the variation. The method differs depending on the purpose. In this research, we adopted MTS which sets homogeneous population as unit space and calculates the degree of variation of object as distance from unit space. In general multivariate analysis, the objective is prediction (determination of normality / abnormality with respect to unknown data), whereas in MTS further selection of items (extraction of effective items for judgment) and accurate cause analysis (abnormality identification of the judged cause item) is possible. Normal distribution is assumed only for the unit space and normal / abnormality is judged by calculating the distance (MD) from the unit space. Furthermore, it is possible to analyze the item that caused the abnormality for the data determined to be abnormal. Using the normal group "unit space" as a criterion, quantitatively judge the sample to be judged (signal data) according to the degree of departure from the unit space. As shown in Fig. 3, if the MD is small, it can be judged as a pattern close to the unit space, and if the MD is large, it can be judged as a pattern far from the unit space [5].

The following points can be cited as features of MTS.

(1) It can judge whether it is normal or abnormal.

Because MD using correlation between variables is used, deviation from unit space can be grasped sensitively.

(2) Can be used for cause analysis.

By using the orthogonal table of 2 level systems, it is possible to estimate the difference of MD between the case of using each variable and the case of not using it, and to identify the deviation, that is, the main cause of abnormality.
Table 3: Unit data

\begin{tabular}{cccccc}
\hline & \multicolumn{5}{c}{ Variable } \\
\cline { 2 - 6 } Sample No. & 1 & 2 & 3 & $\cdots$ & $\mathrm{k}$ \\
\hline 1 & $X_{11}$ & $X_{12}$ & $X_{13}$ & $\cdots$ & $X_{1 k}$ \\
2 & $X_{21}$ & $X_{22}$ & $X_{23}$ & $\cdots$ & $X_{2 k}$ \\
3 & $X_{31}$ & $X_{32}$ & $X_{33}$ & $\cdots$ & $X_{3 k}$ \\
$\vdots$ & $\ldots$ & $\ldots$ & $\cdots$ & $\cdots$ & $\cdots$ \\
$\mathrm{N}$ & $X_{n 1}$ & $X_{n 2}$ & $X_{n 3}$ & $\cdots$ & $X_{n k}$ \\
\hline Average & $\overline{x_{1}}$ & $\overline{x_{2}}$ & $\overline{x_{3}}$ & $\cdots$ & $\overline{x_{k}}$ \\
\hline $\begin{array}{c}\text { Standard } \\
\text { deviation }\end{array}$ & $\rho_{1}$ & $\rho_{2}$ & $\rho_{3}$ & $\cdots$ & $\rho_{k}$ \\
\hline
\end{tabular}

Table 4: Normalized data

\begin{tabular}{cccccc}
\hline & \multicolumn{5}{c}{ Variable } \\
\cline { 2 - 6 } Sample No. & 1 & 2 & 3 & $\cdots$ & $\mathrm{k}$ \\
\hline 1 & $x_{11}$ & $x_{12}$ & $x_{13}$ & $\cdots$ & $x_{1 k}$ \\
2 & $x_{21}$ & $x_{22}$ & $x_{23}$ & $\cdots$ & $x_{2 k}$ \\
3 & $x_{31}$ & $x_{32}$ & $x_{33}$ & $\cdots$ & $x_{3 k}$ \\
$\vdots$ & $\cdots$ & $\cdots$ & $\cdots$ & $\cdots$ & $\cdots$ \\
$\mathrm{N}$ & $x_{n 1}$ & $x_{n 2}$ & $x_{n 3}$ & $\cdots$ & $x_{n k}$ \\
\hline Average & 0 & 0 & 0 & $\cdots$ & 0 \\
\hline Standard & 1 & 1 & 1 & $\cdots$ & 1 \\
deviation & & & & & \\
\hline
\end{tabular}

(3) It can constitute the scale for things that are difficult to quantify.

MD used in MTS can be used as a measure for something hard to express with one numerical value such as difference in appearance and waveform difference.

2.4 Calculation formula of "MTS" In this section, we explain the calculation formula of MTS method.

(1) Unit space data and leveling.

Assume that $k$ variables and $n$ samples are obtained as data in unit space as shown in Table 3. The table also shows the mean value and standard deviation of each variable.

For each variable $x_{j}$, calculate the next normalized value $X_{j}$.

$$
X_{i j}=\frac{x_{i j}-\overline{x_{j}}}{\sigma_{j}} \quad(i=1,2, \cdots, n ; \quad j=1,2, \cdots, k)
$$

Table 4 shows the normalized data again. The average value $X_{j}$ of each variable after the time is 0 and the standard deviation is 1 . The standard deviation $\rho$ in MTS is obtained by the following calculation formula with respect to each variable $x_{j}$ with all data as a population.

That is, the degree of freedom is determined by the total number of data the average value of MD of unit data is 1 . Generally, in calculating the standard deviation, the symbol " $\rho$ " is used as a sign of the population standard deviation, but in this paper it is used as a symbol of the standard deviation to be determined from the data.

$$
\sigma=\sqrt{\frac{1}{n} \sum_{i=1}^{n}\left(x_{i}-\bar{x}\right)^{2}}
$$


(2) Calculation of correlation matrix and inverse matrix.

For the $k$ types of variables in Table 4, calculate the correlation matrix $\boldsymbol{R}$.The correlation matrix is a $k \times k$ square matrix, and the component $r_{i j}$ is a correlation coefficient between the variable $i$ and the variable $j$.

$$
\begin{aligned}
\boldsymbol{R} & =\left[\begin{array}{ccc}
1 & \cdots & r_{1 k} \\
\vdots & \ddots & \vdots \\
r_{k 1} & \cdots & 1
\end{array}\right] \\
r_{i j} & =\frac{\sum\left(X_{p i} \times X_{p j}\right)}{n} \quad(p=1, \cdots, n)
\end{aligned}
$$

Next, calculate the inverse matrix $\boldsymbol{R}^{-1}$ of the correlation matrix $\boldsymbol{R}$, and let it be the matrix $\boldsymbol{A}$.

$$
\boldsymbol{A}=\boldsymbol{R}^{-1}=\left[\begin{array}{ccc}
a_{11} & \cdots & a_{1 k} \\
\vdots & \ddots & \vdots \\
a_{k 1} & \cdots & a_{k k}
\end{array}\right]
$$

(3) MD calculation.

Variable pair of unknown (target) data,

$$
y=\left(y_{1}, y_{2}, \cdots, y_{k}\right)
$$

The MD of this variable group normalizes $y$ to $Y$ according to equation (1),

$$
\begin{aligned}
& \boldsymbol{Y}=\left(Y_{1}, Y_{2}, \cdots, Y_{k}\right) \\
& D^{2}=\frac{\boldsymbol{Y} \boldsymbol{A} \boldsymbol{Y}^{\boldsymbol{T}}}{k}=\sum_{i=1}^{k} \sum_{j=1}^{k} a_{i j} Y_{i} Y_{j} / k
\end{aligned}
$$

As shown in the equation (8), in the MTS method, the distance is divided by the number of items $k$.

The Mahalanobis distance (MD) obtained at this time is the square value $D_{2}$.

\section{Diagnosis and determination}

The standard analysis procedure of MTS is as follows [7].

(1) Determine the unit space according to the item (variable, parameter) that expresses the pattern and the purpose of the analysis and collect the data.

(2) Calculate MD of unit space data.

(3) Calculate MD of evaluation data not belonging to unit space and check whether it is correctly identified as unit space.

(4) Using the orthogonal table, select items (variables, parameters) used for judgment.

(5) Calculate MD of unknown data and judge whether it is normal or abnormal.

(6) If necessary, for the data judged as abnormal, analyze the item which caused the abnormality.

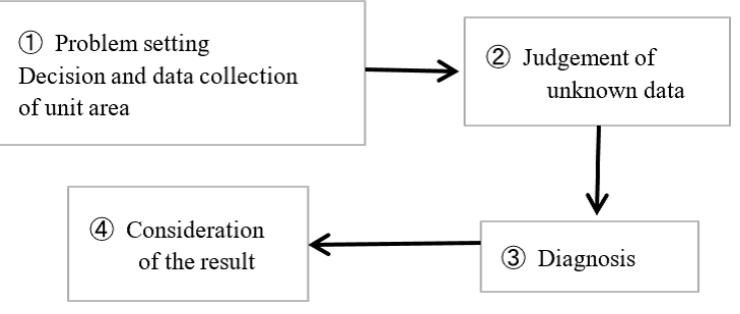

Figure 4: Analysis procedure

The main functions of the software used for the analysis of this study case are as follows. Software name: MTRTAddins (Angle Try Co., Ltd.)

(a) MD calculation of unit space

(b) MD calculation of signal data

(c) Effectiveness analysis (display of analysis results)

(d) Individual diagnosis (contribution degree method)

The analysis procedure of this research is shown in Fig. 4.

3.1 MD calculation of unit and signal data The parameters of the unit space data related to the wind turbine output characteristics are extracted from the 6 items of wind speed, circumferential speed, rotational speed, wind energy and ambient temperature and wind power, and the calculation results of MD are shown in Fig. 5.

The wind turbine output data in the operation start period (Experiment period: 9 months from March 2012 to December 2012) was taken as "unit data" and the output data in the diagnosis target period (Experiment period: 3 months from March 2013 to June 2013) was taken as "signal data". MD calculation results of "Unit data" and "Signal data" are shown in Table 5 and Table 6, respectively. From the calculation result of Fig. 5, the MD mean value of unit data $0.833<1$, the number of normal data 250 , the number of gray data 2 , the number of abnormal data 0 , and the validity of the selected unit data was confirmed.

In addition, the MD average value of the signal data was $1.447>1$, the number of normal data was 45 , the number of gray data was 2 , and the number of abnormal data was 2 , and deviations from the unit space of the MD value considering the correlation were confirmed in the wind turbine output characteristics. The MD threshold value in this case was set to 4.0.

3.2 Effectiveness analysis The effectiveness analysis is a process of analyzing which parameter is effective for abnormality detection. In this research, we adopted the method of "fusion evaluation characteristics" by SN ratio (Signal to Noise ratio) and MD value for discrimination of pass / fail in pattern recognition system [3] [6].

The $\mathrm{SN}$ ratio is an evaluation scale of the functionality used in quality engineering. In MTS, the pattern recognition accuracy depends on the definition items of unit space, the definition of items (variables), and further the measurement method. For this reason, the quality is evaluated using 


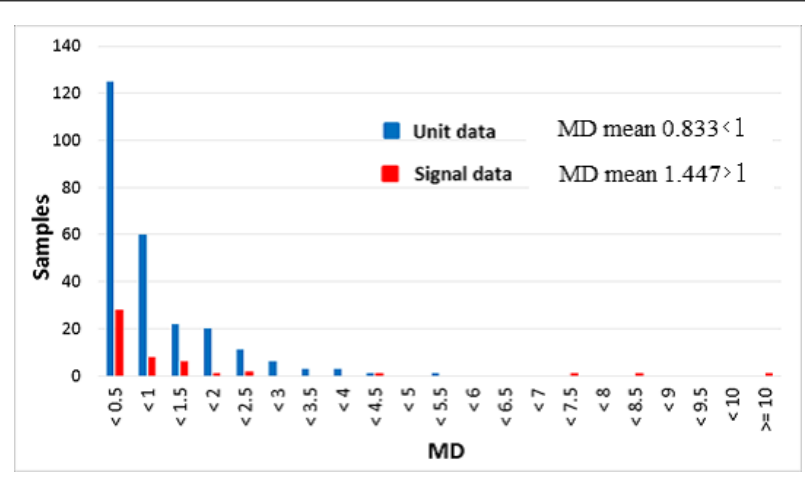

Figure 5: Calculation result of MD

Table 5: Unit data

\begin{tabular}{|c|c|c|c|}
\hline No. & MD value & MD & Frequency \\
\hline 1 & 2.445098 & $<0.5$ & 125 \\
\hline 2 & 1.958154 & $<1$ & 60 \\
\hline 3 & 1.533857 & $<1.5$ & 22 \\
\hline 4 & 1.877757 & $<2$ & 20 \\
\hline 5 & 1.593308 & $<2.5$ & 11 \\
\hline 6 & 1.494618 & $<3$ & 6 \\
\hline 7 & 1.970119 & $<3.5$ & 3 \\
\hline 8 & 2.194204 & $<4$ & 3 \\
\hline 9 & 1.958154 & $<4.5$ & 1 \\
\hline 10 & 2.2653 & $<5$ & 0 \\
\hline 11 & 1.505382 & $<5.5$ & 1 \\
\hline 12 & 1.644807 & $<6$ & 0 \\
\hline 13 & 1.554838 & $<6.5$ & 0 \\
\hline 14 & 1.669784 & $<7$ & 0 \\
\hline 15 & 1.809105 & $<7.5$ & 0 \\
\hline 16 & 1.700057 & $<8$ & 0 \\
\hline 17 & 1.910132 & $<8.5$ & 0 \\
\hline 18 & 1.927801 & $<9$ & 0 \\
\hline 19 & 2.7488 & $<9.5$ & 0 \\
\hline 20 & 2.532284 & $<10$ & 0 \\
\hline 21 & 2.078277 & $>=10$ & 0 \\
\hline$\downarrow$ & $\downarrow$ & \multirow{3}{*}{\multicolumn{2}{|c|}{ MD mean $=0.833$}} \\
\hline 58 & 0.483268 & & \\
\hline 59 & 1.424108 & & \\
\hline 60 & 03572993 & \multicolumn{2}{|c|}{ Number of samples : 252} \\
\hline 61 & $\dagger 4.4213$ & \multirow{4}{*}{\multicolumn{2}{|c|}{$\begin{array}{c}\text { Number of Nomal data : } 250 \\
\dagger \quad \text { Number of gray data : } 2 \\
\pm \quad \text { Number of abnormal data : } 0\end{array}$}} \\
\hline$\downarrow$ & $\bar{\downarrow}$ & & \\
\hline 108 & 0.242303 & & \\
\hline 109 & 2.042718 & & \\
\hline 110 & +5.106363 & & \\
\hline$\downarrow$ & $\bar{\downarrow}$ & & \\
\hline 247 & 0.327734 & & \\
\hline 248 & 0.253915 & & \\
\hline 249 & 0.69052 & & \\
\hline 250 & 0.78157 & & \\
\hline 251 & 0.399354 & & \\
\hline 252 & 0.432948 & & \\
\hline
\end{tabular}

the SN ratio Quality engineering has the concept of various $\mathrm{SN}$ ratios, but there are the following two methods for calculating the $\mathrm{SN}$ ratio used in MTS.

(a) If it is difficult to give a numerical value to the degree of abnormality, apply the SN ratio of the "Preferably large characteristics".
Table 6: Signal data

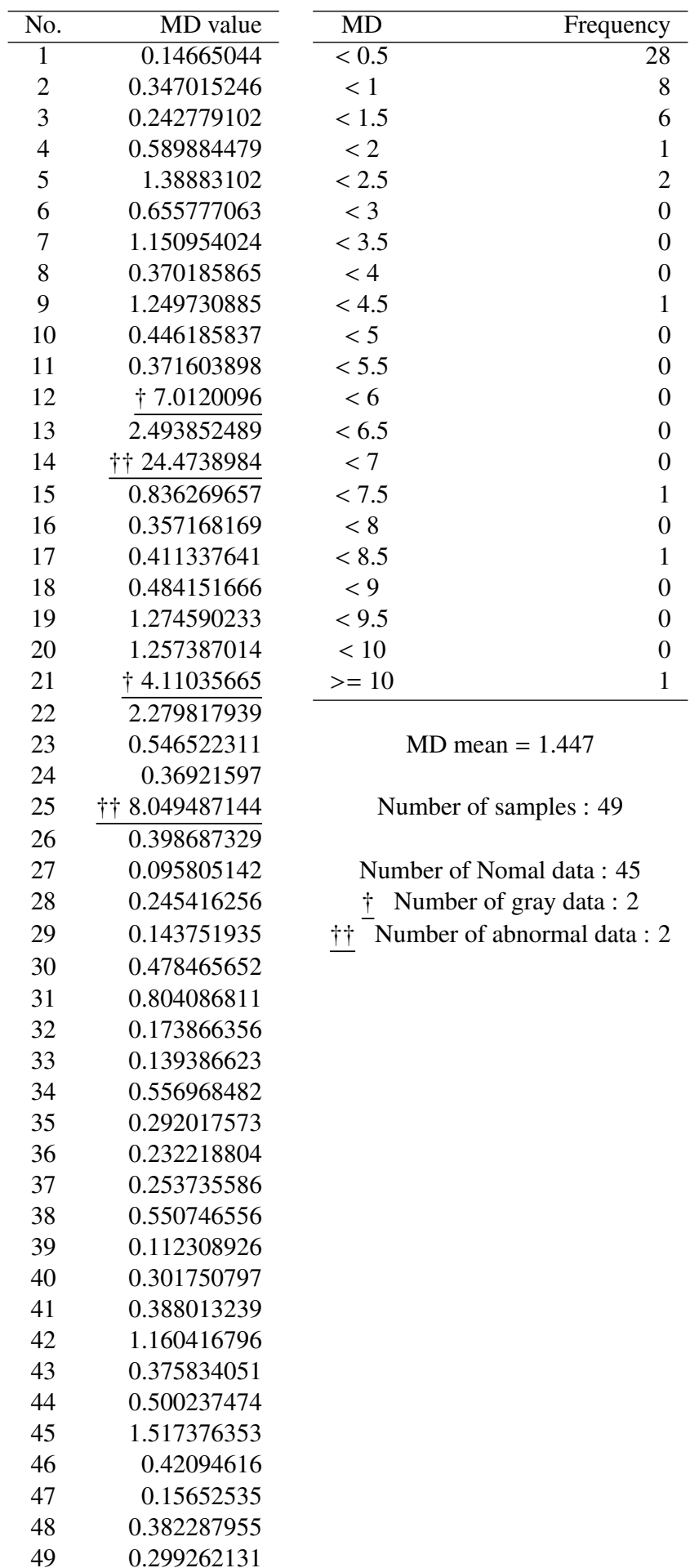

(b) When the degree of abnormality can be quantified, the SN ratio of the "dynamic characteristics" is applied.

It is desirable that the MD value of data known not to belong to the unit space be as large as possible.

In quality engineering, "larger desirable properties" are called "Preferably large characteristics". Since there are generally variations when there are many data, the $\mathrm{SN}$ ratio is to quantify desirability collectively including variations. 


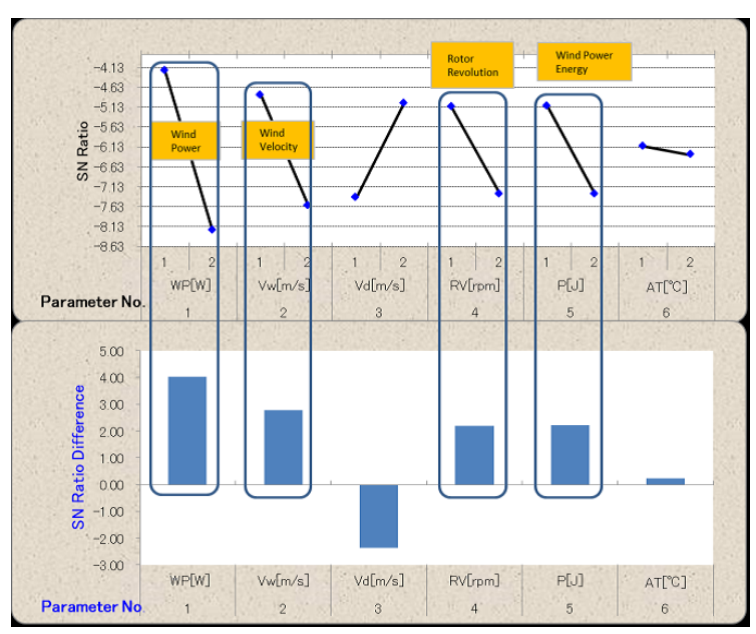

Figure 6: Effectiveness analysis result

Therefore, in the analysis in this research, "S / N ratio of the preferably large characteristics" and an orthogonal table were applied.

The calculation formula of the SN ratio $(\eta)$ of the preferably large characteristics is as shown in the formula (9). At this time, it is better that the $\mathrm{SN}$ ratio is large. Here, $D_{2}$ is the Mahalanobis distance (the value of the square), and $k$ is the number of signal data not belonging to the unit space.

$$
\eta=-10 \log \left\{\frac{\left.\left(1 / D_{1}^{2}+1 / D_{2}^{2}+\cdots+1 / D_{k}^{2}\right)\right)}{k}\right\}
$$

The analysis result is shown in Fig. 6. The vertical axis of the graph represents the $\mathrm{SN}$ ratio of the level of the orthogonal table, and the vertical axis of the graph in the lower part represents the difference in $\mathrm{SN}$ ratio for each parameter level. In the graph below, it means that the items are larger in the positive direction, and in the upper graph, the items indicating the large downward slope are effective items for judgment of abnormality. Also, there is an item with a negative value in the graph below, which means "the detection sensitivity is lowered by using that item". It is judged that the effectiveness is larger as the items with larger degree of downward slope (windmill output, wind speed, rotor revolution, wind energy) [9] [10].

\subsection{Individual diagnosis "Individual diagnosis" is a} process of calculating the degree of contribution to the MD value for each item. It is calculated for each individual signal data and has meaning only when the MD is large. An analysis result of individual diagnosis is shown in Fig. 7. The horizontal axis of Fig. 7 shows the parameter number (refer to the correspondence table of parameter No. and name).

The value of the contribution degree represents the SN ratio of the parameter having a large influence on the MD value. ( $c f$. Expression 9)

Individual diagnosis has meaning in case of abnormality, so it can be confirmed that in the wind turbine output characteristics, the items with a large contribution to the MD value are the wind speed, the rotor rotation speed, and the

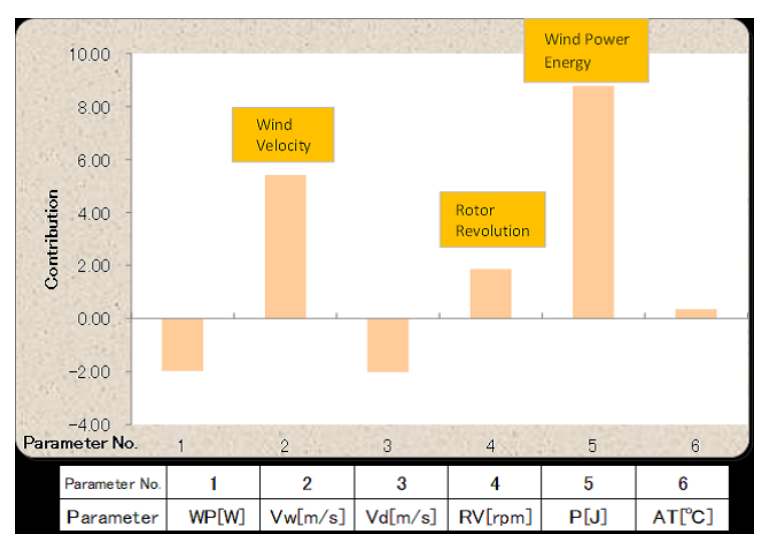

Figure 7: Individual diagnosis result

wind energy. In other words, it can be said that the degree of influence on departure from unit data is large [14].

\section{Output characteristics and high efficiency}

With respect to variations in output characteristics of wind turbines, we will focus on the three items of wind speed, rotor speed, and wind energy from the results of cause analysis and diagnosis by MTS (effectiveness analysis, individual diagnosis), and the technical views are described below.

The output coefficient $C_{p}$ is used as an index indicating how much power can be obtained from the power of the wind. This output coefficient $C_{p}$ is expressed by the ratio of wind turbine output to wind power (Expression 10). In addition, the peripheral speed ratio $\lambda$ is defined as the ratio of the peripheral speed of the wind turbine propeller to the wind speed (Expression 11).

$C_{p}=\frac{W_{p}}{\frac{1}{2} \rho A V_{W}^{3}}$

$W_{p}$ : Power to obtain a windmill [J]

$\rho:$ Air density $\left[\mathrm{kg} / \mathrm{m}^{3}\right]$

$A$ : Wind receiving area $\left[\mathrm{m}^{2}\right]$

$V_{W}:$ Wind velocity $[\mathrm{m} / \mathrm{s}]$

$\lambda=\frac{\omega R}{V_{W}}=\frac{2 \pi R n}{V_{W}}$

$\omega$ : Rotor angular velocity $[1 / \mathrm{s}]$

$R$ : Rotor radius [m]

$n$ : Wind turbine speed [rps]

$W_{p}=Q \omega$

$Q:$ Torque $[\mathrm{N} \cdot \mathrm{m}]$

From the theoretical formula, the windmill means that there exists an optimum rotation speed at which maximum power can be obtained depending on the wind speed. Therefore, it can be said that it is preferable to control the wind power generator by selecting the rotation speed at which the 


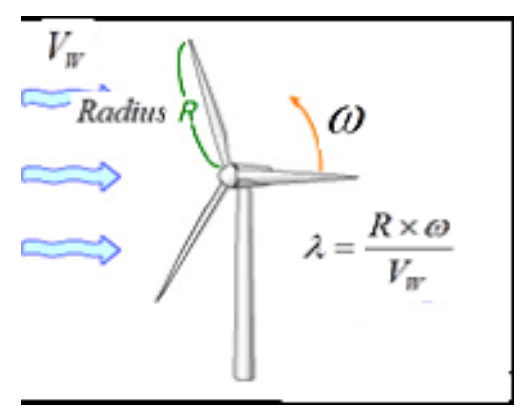

Figure 8: Windmill output model

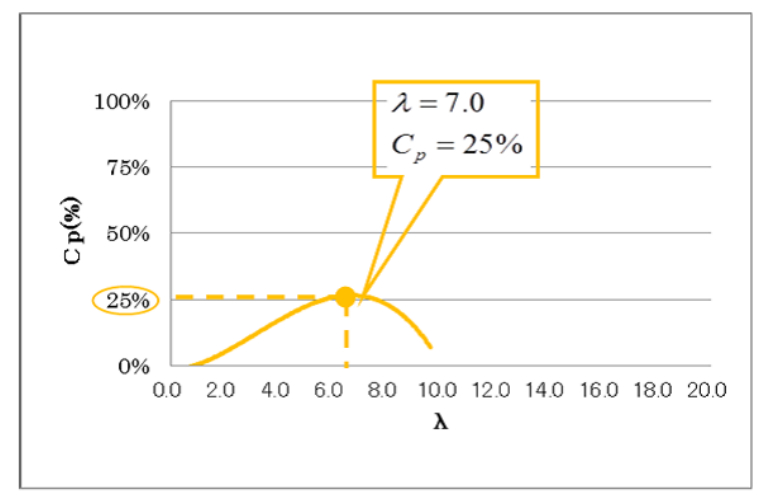

Figure 9: Relations of $C_{p}$ and $\lambda$

maximum output is obtained for each wind speed in order to obtain a higher efficiency and obtain a large amount of power generation.

Fig. 8 shows the wind turbine output model.

The results of analyzing the relationship between $C_{p}$ and $\lambda$ in this study are shown in Fig. 9 (Approximate curve). It is a curve with the maximum point $\left(\lambda=7.0, C_{p}=25 \%\right.$ at $V_{W}=12.5 \mathrm{~m} / \mathrm{s}$ ) as shown in Fig. 9 .

It means that there is a rotation speed of the wind turbine propeller which is optimum for a certain wind speed. That is, when the peripheral speed ratio $\lambda$ is 7.0 and the actual output coefficient $C_{p}$ is $25 \%$, the operation efficiency is low. In the case of a propeller type in general, it is about $45 \%$ at maximum [12] [13].

In addition, the peripheral speed ratio can be confirmed from Fig. 10, as the propeller radius $R$ is constant, the ratio is determined by the wind speed and the angular velocity, and the relationship between the wind speed and the rotor speed influences the dispersion of wind turbine output characteristics.

The wind turbine has the optimum rotation speed that can obtain the maximum output depending on the wind speed. Therefore, in order to efficiently obtain more electric power, high efficiency operation can be realized by controlling the generator so as to achieve the optimum generator rotation speed to make the wind turbine follow the optimum rotation speed. In this study, the ideal rotation speed and reduction ratio were obtained by the following calculation formula.

Ideal rotation speed: $N_{e}$,

$$
N_{e}=\frac{1000 \mathrm{rpm} \times 1.5 \mathrm{~m} / \mathrm{s}}{12.5 \mathrm{~m} / \mathrm{s}}=840 \mathrm{rpm}
$$

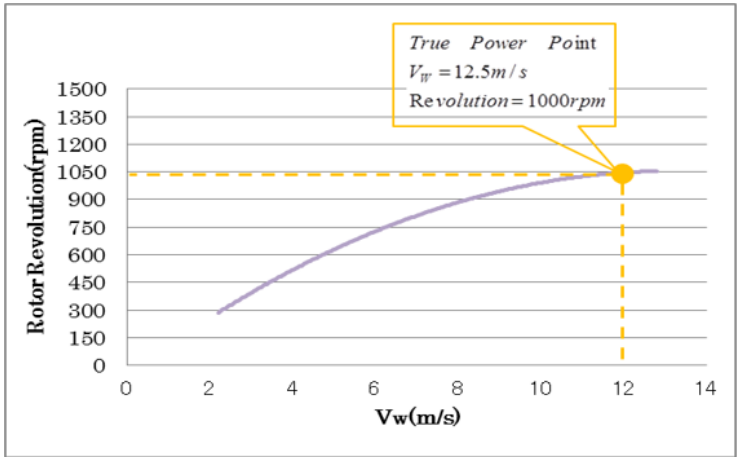

Figure 10: Relations of $V_{w}$ and $R_{v}$

Reduction ratio: $i$,

$$
i=\frac{840}{1000}=0.84 \fallingdotseq \frac{1}{1.2}
$$

The ideal rotational speed $N_{e}$ of the equation (13) is the calculation result when obtaining the maximum efficiency from the analysis result of Fig. 12 (wind speed $V_{w}=10.5$ $\mathrm{m} / \mathrm{s})$.

On the basis of the above calculation results, a deceleration device by inverter control was installed in the motor of the actual machine, and evaluation on the output characteristics were carried out.

\section{Conclusion}

In this research, the factors that cause the wind turbine output characteristics in the compact wind power generation system were analyzed by MTS and the causal parameters were extracted from the analysis results obtained. Considering from the result, "When the wind turbine propeller is rotating at a constant speed, as the wind speed changes, the circumferential speed ratio also changes, so it will deviate from the maximum output point, and wind energy cannot be extracted to the maximum [8] [11].

Therefore, by changing the wind turbine propeller according to wind fluctuation and keeping the peripheral speed ratio $\lambda$ constant so that the maximum value of the output coefficient $C_{p}$ is maintained, the wind energy is converted to the maximum power energy it will be possible was obtained.

Fig. 11 shows the actual measurement result of the output characteristics by installing a speed reducer by inverter control in the motor of the actual machine (Experiment period: 3 months from March 2015 to June 2015).

Fig. 12 shows the actual analysis results of wind speed $V_{w}$ and output coefficient $C_{p}$ (Approximate curve). $C_{p}$ at a wind speed of $10.5 \mathrm{~m} / \mathrm{s}$ was $40 \%$, and the conversion efficiency of wind energy was improved.

From the actual measurement results, the standard output and the actual output were almost the same value (within \pm $3 \%$ ) in the range of the power output from the wind speed $10.5 \mathrm{~m} / \mathrm{s}$ to $12.5 \mathrm{~m} / \mathrm{s}$, and the output characteristics and efficiency stabilization were achieved. However, in the rotational speed control operation at the maximum output point, research on the influence on the responsiveness by the inertial force of the wind turbine propeller is a future task. 


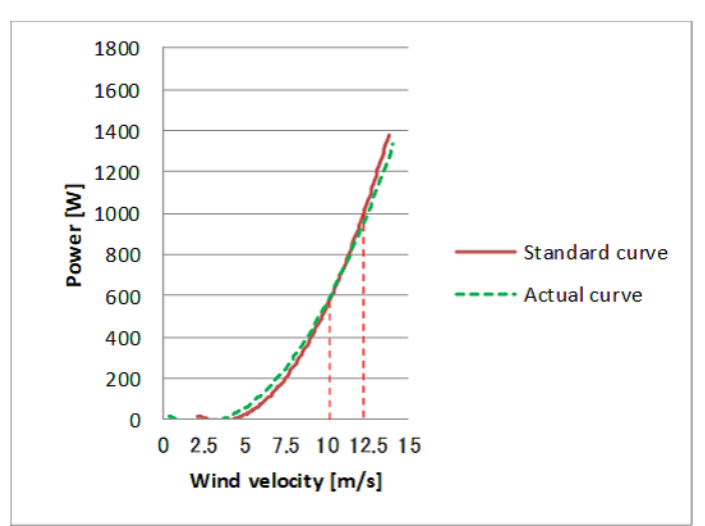

Figure 11: Actual output curve

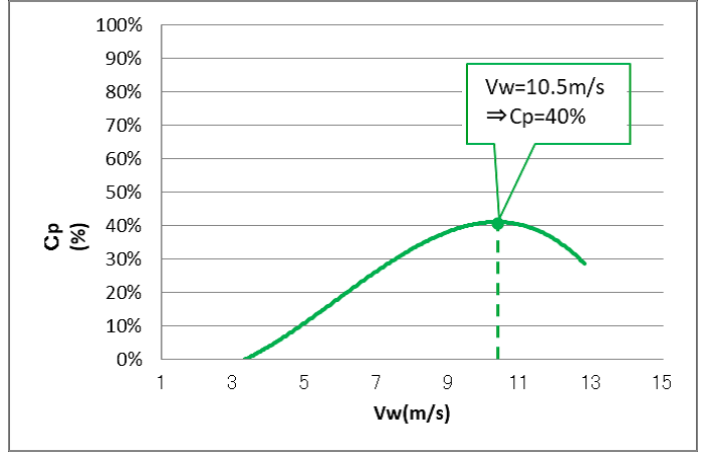

Figure 12: Actual relation of $C_{p}$ and $V_{w}$

\section{References}

[1] Y. Shimizu, "Wind-generated electricity technology" (revised edition), power company, pp.25-56, 1999.

[2] General corporation energy resources official journal of a scientific society, "Energy resources", vol.33, no .3, 2012.

[3] K. Tatebayashi and S. Tejima, "Introduction MT system", Federation of Japanese technology, pp.33-57, 2012.

[4] K. Nakata, "Pattern recognition and application", Corona company, pp.43-80, 1990.

[5] H. Yano, "Technology development of the information design with the computer- Simulation and MT system", Japan Standards Association, pp.310-368, 2004.

[6] S. Koshimizu and M. Suzuki, "Practice Quality Engineering to acquire by experience by a Virtual Experiment", Daily industry newspaper publisher, pp.67-89, 2007.

[7] Y. Watanabe, "Practice Taguchi Methods", Federation of Japanese technology, pp.12-18, 2006.

[8] H. Yano, "Introduction to Quality Engineering numeration", Japan Standards Association, pp.271-286, 2002.

[9] T. Mori, "Application and Mathematics of the Taguchi Methods", Optimization engineering using the Taguchi Methods, trendy book, pp.323-338, 2005.

[10] Gennichi Taguchi, Quality Engineering lecture no.5 "Quality Engineering Casebook - Japan public", Japan Standards Association, pp.1-12, 1992.
[11] Y. Hasegawa, "Taguchi method solves environmental problems", Japan Science and Technology Publishing, pp.175193, 2013.

[12] I. Ushiyama, "Wind energy reader", Ohmsha, pp.147, 2005.

[13] I. Ushiyama, "New energy to think thoroughly", Mainichi Newspapers, pp.86-90, 2011.

[14] G. Taguchi, "Quality Engineering Applied Course Technology Development of Information Design by Computer Simulation and MT System", Japan Standards Association, pp.127-129, 2004.

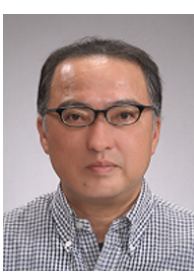

Eiji Toma (Member) was born in Hokkaido, Japan, on December 17, 1959. When he was engaged as a manufacturing engineer at an automobile parts manufacturer, he acquired a "Professional Engineer (P.E.jp)" and "APEC engineer" in 2018, and is presently an professor at National Institute of Technology, Tomakomai College. He has worked on research of the quality engineering. He is member of IIAE, JSME, JSDE, JSAE, RQES, IPEJ.

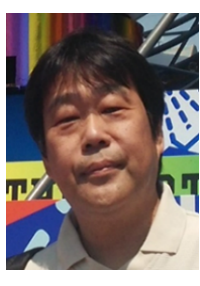

Hiroshi Tanaka (Non-member) is a Professor in the Department of Mechanical Engineering at Aichi Institute of Technology, Japan. He received his Ph.D from Nagoya University and worked as a process engineer in automobile parts company for 20 years. His research interests include environment friendly manufacturing process and quality engineering.

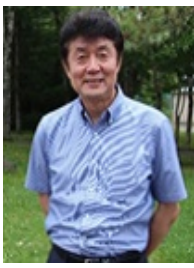

Kazushige Kikuta (Non-member) is a professor in the Department of Mechanical Engineering at National Institute of Technology, Tomakomai College, Japan. He received his Ph.D from Hokkaido University and his current research activity is the energy management system construction for a house and a building in the cold climate region. 